\title{
Fatores de risco cardiovascular em residentes de um hospital universitário
}

As doenças cardiovasculares configuram-se como um problema de saúde pública mundial e são responsáveis por um elevado número de invalidez, resultando em uma necessidade maior de cuidado familiar e prejuízo financeiro ao setor da saúde. Existem fatores determinantes para o aparecimento dessas doenças, que podem ser modificáveis ou não. Assim sendo, esse projeto teve como objetivo identificar os fatores de risco cardiovascular nos estudantes do programa de residência multiprofissional de um hospital universitário do município de Manaus-AM. Tratou-se de um estudo descritivo, transversal, de campo, com abordagem quantitativa realizada em um Hospital Universitário, aprovado pelo Comitê de Ética e Pesquisa, incluiu 85 residentes da Universidade Federal do Amazonas (UFAM). Foram identificados alguns fatores de risco para doença cardiovascular. Com os resultados, percebe-se a necessidade de orientações e ações efetivas que visem à saúde desta população, através do cuidado.

Palavras-chave: Enfermagem; Doenças Cardiovasculares; Saúde do Trabalhador

\section{Cardiovascular risk factors in residents of a university hospital}

Cardiovascular diseases are configured as a worldwide public health problem and are responsible for a high number of disabilities, resulting in a greater need fo family care and financial loss to the health sector. There are determining factors for the appearance of these diseases, which can be modified or not. Therefore, this project aimed to identify cardiovascular risk factors among students in the multiprofessional residency program at a university hospital in the municipality of Manaus- AM. It was a descriptive, cross-sectional, field study, with a quantitative approach carried out in a University Hospital, approved by the Ethics and Research Committee, included 85 residents of the Federal University of Amazonas (UFAM). Some risk factors for cardiovascular disease have been identified. With the results, there is a need for guidance and effective actions aimed at the health of this population, through care.

Keywords: Nursing; Cardiovascular Diseases; Occupational Health

Topic: Enfermagem Geral

Received: $14 / 06 / 2021$

Approved: 11/07/2021

Reviewed anonymously in the process of blind peer.

Renan Sallazar Ferreira Pereira

Universidade Federal do Tocantins, Brasil http://lattes.cnpq.br/8154326371029706 http://orcid.org/0000-0001-5140-4046 renansallazar@gmail.com

Thiago do Nascimento Reis (iD)

Universidade Federal do Amazonas, Brasil http://lattes.cnpq.br/7019954711183231 http://orcid.org/0000-0002-7437-6305 acad.thiagoreis25@gmail.com
Universidade Federal do Amazonas, Brasil

http://lattes.cnpq.br/9019612448128331

http://orcid.org/0000-0001-8553-7269

laiufam@gmail.com

Albert Einstein Tavares de Seixas (D)

Universidade Federal do Amazonas, Brasil http://lattes.cnpq.br/6604974384424214 http://orcid.org/0000-0002-9363-762X albertt.einstein19@gmail.com http://lattes.cnpq.br/7449173955289609 http://orcid.org/0000-0002-1329-0612

driellygalvao07@hotmail.com

Everton de Oliveira Pinto (iD)

Universidade Federal do Amazonas, Brasi http://lattes.cnpq.br/7587189002602696 http://orcid.org/0000-0003-3977-273X everton.oliveira-@hotmail.com

Karoline Costa de Souza (iD)

Universidade Federal do Amazonas, Brasi http://lattes.cnpq.br/3136475385980110 http://orcid.org/0000-0002-3547-8472 karolinecosta.d@gmail.com

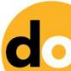

DOI: 10.6008/CBPC2236-9600.2021.003.0005
Referencing this:

SOUSA, S. O.; FERREIRA, A. A.; SEIXAS, A. E. T.; GALVÃO, D. S.; PINTO, E. O.; SOUZA, K. C.; PEREIRA, R. S. F.; REIS, T. N.. Fatores de risco cardiovascular em residentes de um hospital universitário. Scire Salutis, v.11, n.3, p.28-37, 2021. DOI:

http://doi.org/10.6008/CBPC2236-9600.2021.003.0005 


\section{INTRODUÇÃO}

As Doenças Cardiovasculares (DCV) representam um dos maiores problemas de saúde pública devido às taxas elevadas de morbidade e mortalidade na população, além dos transtornos decorrentes da invalidez que grande parte dos indivíduos acometidos por tais problemas passam a enfrentar ${ }^{1}$. No Brasil as DCV são responsáveis pela maior taxa de mortalidade na população, estando presente em $30 \%$ dos casos, ou seja, 17 milhões de pessoas morreram em decorrência de $\mathrm{DCV}^{2,3}$. Autores ${ }^{4}$ afirmam que o ônus a economia é proveniente também da diminuição da força de trabalho, pois os indivíduos que ficam sequelados das DCV passam a não exercer mais a profissão a qual exerciam.

A estimativa é de que o maior número de mortes no mundo, em 2030 , seja por DCV 5 . A Hipertensão Arterial Sistêmica (HAS) e Diabetes Mellitus (DM) são as principais causas de DCV, um estudo conduzido nos Estados Unidos da América deu conta que a HAS esteve presente em $69 \%$ dos casos de Infarto Agudo do Miocárdio (IAM), 77\% de Acidente Vascular Encefálico (AVE), 75\% dos casos de Insuficiência Cardíaca (IC) e 60\% dos casos de Doença Arterial Periférica (DAP). A associação de HAS com DM implica em fatores de alto risco de DCV e seus impactos na economia são elevados, além das perdas produtivas e rendas familiares ${ }^{4}$.

Existem fatores determinantes para o aparecimento de DCV, incluindo fatores metabólicos, sociais e um dos mais importantes, pois são modificáveis - os comportamentais. Dentro dessa última classe estão inseridos o tabagismo, a má alimentação, uso abusivo do álcool e ausência de atividade física ${ }^{6}$.

Neste contexto, o referido estudo investigou quais os fatores de risco cardiovascular os residentes estão susceptíveis. Tendo em vista que as identificações desses fatores servirão para melhor compreender o panorama de vida desses residentes, assim permitindo que haja melhor adequação e/ou meios que visem reduzir e/ou evitar a manutenção de condições que impliquem em prejuízos à saúde desses residentes.

\section{METODOLOGIA}

Trata-se de um estudo descritivo, transversal, de campo, com abordagem quantitativa. O referido estudo foi realizado em um hospital universitário que atualmente dispõe de estrutura de 48 consultórios e 159 leitos hospitalares, caracterizando-se como um hospital de grande porte, conduzido com residentes dos Programas de Residência de uma instituição pública.

Todos aqueles que aceitaram participar do estudo tiveram acesso primeiramente ao Termo de Consentimento Livre e Esclarecido - TCLE, assinando em duas vias, que visava esclarecer dúvidas e proteger o sujeito do estudo, mantendo a identidade em sigilo, proporcionando segurança também para o pesquisador, que por este meio manifesta respeito à ética durante o desenvolvimento do trabalho. 0 mesmo foi elaborado conforme a Resolução 466/12, que dispõe sobre os direitos e deveres que devem ser assegurados à pessoa que se dispõe a participar de uma pesquisa que envolve seres humanos.

Incluiu-se residentes que estivessem realizando suas atividades referentes ao programa no período de coleta de dados, com idade igual ou superior a 18 anos, de qualquer período da residência. Foram excluídos deste estudo residentes em período gestacional. 
Para a coleta de dados foram utilizados dois questionários e três formulários. Os instrumentos foram aplicados pela pesquisadora e 2 acadêmicos, que passaram por treinamento para realizarem a coleta de dados. Realizou-se a coleta em um local tranquilo e reservado, cedido pela coordenação após as reuniões semanais da residência, conforme o calendário da residência já acordado. Ou em uma sala reservada da unidade onde exerciam suas funções.

Inicialmente a amostra seria de 151 entrevistados da Residência Médica e 50 da Residência Multiprofissional. No entanto, foram coletados os dados com 50 profissionais médicos e 35 das demais profissões. Representando assim 85 coletas do total de 151. Desses, 2 foram retirados após aplicação de critérios de exclusão, 1 por ter trancado a residência e 3 por recusarem participar da pesquisa. Os outros 110 residentes foram os que não retornaram contato algum via e-mail, aplicativo de mensagens e nenhuma outra abordagem realizada pela pesquisadora. Todos foram contactados mais de duas vezes, seja por email, aplicativo e outros meios de comunicação, sendo as mensagens ignoradas e os e-mails não respondidos, uma vez que a pesquisadora não teve acesso às suas escalas de trabalho e nem à unidade em que atuavam.

O APÊNDICE A é constituído de três partes, sendo a primeira (parte A) relativa aos dados pessoais, a segunda (parte B) aos dados profissionais, a terceira (parte C) aos dados sobre o estilo de vida dos trabalhadores. No APÊNDICE B constam os dados sobre os fatores de risco, como o uso de anticoncepcional, antecedentes familiares para HAS, IAM, AVC, DM, hipercolesterolemia, angina pectoris e outras doenças e morbidade referida, como história clínica para HAS, DM, hipercolesterolemia e estresse.

O Alcohol Use Disorders Identification Test (AUDIT - ANEXO A) visa rastrear o uso excessivo de álcool; foi criado por Piccinelli e é considerado, atualmente, o melhor método para a rastreamento e estratificação do alcoolismo7. O APÊNDICE C compreende os dados antropométricos como: peso, altura, pressão arterial sistólica e diastólica, circunferência abdominal e do pescoço. O ANEXO B fez a Avaliação do Estresse, através do Questionário de Vulnerabilidade ao Estresse.

As medidas foram realizadas com aparelho automático oscilométrico validado. A pressão arterial foi medida por três vezes consecutivas, com intervalo de 1 minuto entre cada medida, na posição sentada, bexiga vazia e após cinco minutos de descanso em ambiente calmo e com temperatura agradável. Foi utilizado manguito com bolsa de borracha de tamanho adequado à circunferência do braço do indivíduo. Para classificação da pressão arterial e medida, foram consideradas as normas apresentadas na VII Diretriz Brasileira de Hipertensão4.

O índice de massa corporal (IMC) foi calculado pela fórmula "peso dividido por estatura ao quadrado", observando as medidas do peso em quilogramas e a da estatura em metros, ou seja, IMC=Peso atual $(\mathrm{Kg})$ / [altura $(\mathrm{m})$ ]. As medições foram realizadas em balança digital. Na ocasião, os indivíduos foram orientados a trajar roupas leves e retirarem os sapatos. Além disso, foi necessário que os mesmos ficassem eretos na posição ortostática, com os braços ao longo do corpo, os pés unidos e a cabeça erguida com olhos fixos no horizonte. O IMC foi classificado conforme preconizado ${ }^{8}$.

A circunferência abdominal foi medida por meio de fita milimetrada flexível, no ponto médio entre 
o rebordo costal inferior e a crista ilíaca. Foram considerados valores anormais aqueles superiores a 102 centímetros para homens e 88 centímetros para mulheres.

Considera-se tabagista aquele indivíduo que consome regularmente, no mínimo, um cigarro por dia, durante um ano ou que tenha fumado no ano anterior a participação neste estudo. Ex-fumantes são aqueles que não fumaram no ano anterior a inclusão neste estudo, mas que o tenham feito entre um e 10 anos anteriores à pesquisa.

Os dados obtidos foram apresentados utilizando-se um banco de dados criado em planilha no programa de computador Excel 2010, apresentados na forma de tabelas e figuras e analisados quantitativamente. Após a verificação dos dados objetivos e subjetivos, os mesmos foram postos no programa de estatística denominado IBM - SPSS Statistics ${ }^{\circledR}$ versão 21 no qual foi realizada a análise. As variáveis contínuas foram apresentadas descritivamente em média e desvio-padrão, valores mínimos e máximos e as variáveis categóricas em número absoluto e/ou frequência relativa.

Para análise das variáveis categóricas foi utilizado o Teste Qui-quadrado e para as variáveis contínuas o Teste $\mathrm{t}$ de Student, Teste $\mathrm{U}$ de Mann-Whitney ou o Teste de Kruskal-Wallis, quando pertinentes. Para comparar as médias dos dados contínuos foi usada a análise de variância. Foi aceito como significativo $\mathrm{P} \leq 0,05$. A análise multivariada em relação à prevalência dos fatores de risco foi feita a partir das variáveis que apresentassem significância estatística $(p \leq 0,05)$ no modelo univariado.

\section{RESULTADOS E DISCUSSÃO}

Tabela 1: Apresentação dos dados sociodemográficos, hábitos e estilo de vida dos participantes do estudo, ManausAM, 2019

\begin{tabular}{|c|c|c|}
\hline \multirow[t]{2}{*}{ Variáveis } & \multicolumn{2}{|c|}{ Total $=85$} \\
\hline & $\mathbf{N}$ & $\%$ \\
\hline \multicolumn{3}{|l|}{ Sexo } \\
\hline Masculino & 27 & $31,8 \%$ \\
\hline Feminino & 58 & $68,2 \%$ \\
\hline \multicolumn{3}{|l|}{ Idade } \\
\hline Média $\pm d p$ & $28,41 \pm 4,63$ & \\
\hline \multicolumn{3}{|l|}{ Raça/cor } \\
\hline Branco & 30 & $35,3 \%$ \\
\hline Preto & 3 & $3,5 \%$ \\
\hline Pardo & 44 & $51,8 \%$ \\
\hline Amarelo & 1 & $1,2 \%$ \\
\hline Ignorado & 7 & $8,2 \%$ \\
\hline \multicolumn{3}{|l|}{ Estado civil } \\
\hline Solteiro & 64 & $75,3 \%$ \\
\hline Casado & 19 & $22,4 \%$ \\
\hline União estável & 2 & $2,4 \%$ \\
\hline \multicolumn{3}{|c|}{ Pratica de exercícios regularmente } \\
\hline Sim & 38 & $44,7 \%$ \\
\hline Não & 45 & $52,9 \%$ \\
\hline Ignorado & 2 & $2,4 \%$ \\
\hline \multicolumn{3}{|c|}{ Tipo de exercício que pratica } \\
\hline Caminhada/corrida & 7 & $8,2 \%$ \\
\hline Ciclismo & 1 & $1,2 \%$ \\
\hline Musculação & 15 & $17,6 \%$ \\
\hline Dança & 2 & $2,4 \%$ \\
\hline Alongamento & 1 & $1,2 \%$ \\
\hline Boxe & 1 & $1,2 \%$ \\
\hline Funcional & 1 & $1,2 \%$ \\
\hline
\end{tabular}

A tabela 1 mostra os dados dos participantes referente a condição sociodemográfica e hábitos e estilo de vida. O estudo envolveu 85 participantes, sendo a maioria $(68,2 \%)$ do sexo feminino, o sexo 
masculino representou $(31,8 \%)$, a média de idade foi de $(28,41 \pm 4,63)$ anos. Quanto à raça dos participantes, verifica-se que mais da metade $(51,8 \%)$ se declarou ser pardo, seguido dos brancos $(35,3 \%)$, os demais representaram (4,7\%). $75,3 \%$ dos participantes eram solteiros, os casados foram os segundos mais comuns com (22,4\%). Em relação ao fumo, $(91,8 \%)$ declarou nunca ter feito uso de cigarro, enquanto $(8,2 \%)$ referiu uso do mesmo, porém já não o utilizam (tabela 1 ).

De acordo com autores ${ }^{9}$, foi identificada a associação estatística entre o setor de atuação, formação e o uso do tabaco entre os profissionais. Fatores como dependência e desmotivação são responsáveis pela dificuldade de interrupção do uso de cigarro e devido a isso, os profissionais necessitam de acompanhamento para que sejam prevenidas as complicações decorrentes do tabagismo.

Dos participantes, apenas $(44,7 \%)$ praticava alguma atividade física regularmente, enquanto $(52,9 \%)$ não realizava nenhuma. Dentre as atividades, a musculação foi a mais comum $(17,6 \%)$, a caminhada ou corrida foi a segundo mais comum $(8,2 \%)$, no que diz respeito a frequência dessas atividades, $(21,2 \%)$ praticava até 2 vezes por semana, $(15,3 \%)$ praticava por mais de 3 vezes por semana. Os hábitos e estilos de vida adotados por estudantes comumente corroboram para os agravos das complicações inerentes ao sistema cardiovascular ${ }^{10}$.

Tabela 2: Apresentação dos dados referentes ao trabalho dos participantes deste estudo, Manaus-AM, 2019

\begin{tabular}{|c|c|c|}
\hline \multirow[b]{2}{*}{ Variáveis } & \multicolumn{2}{|c|}{ Total=85 } \\
\hline & $\mathbf{N}$ & $\%$ \\
\hline \multicolumn{3}{|l|}{ Local de trabalho } \\
\hline HUGV & 65 & $76,5 \%$ \\
\hline Unidade Básica de Saúde & 4 & $4,7 \%$ \\
\hline Hospital e Pronto Socorro & 1 & $1,2 \%$ \\
\hline Maternidade & 8 & $9,4 \%$ \\
\hline Patologia & 1 & $1,2 \%$ \\
\hline DPML & 2 & $2,4 \%$ \\
\hline Ambulatório & 1 & $1,2 \%$ \\
\hline Ignorado & 3 & $3,5 \%$ \\
\hline \multicolumn{3}{|l|}{ Profissão } \\
\hline Enfermeiro & 17 & $20,0 \%$ \\
\hline Médico & 51 & $60,0 \%$ \\
\hline Fisioterapeuta & 5 & $5,9 \%$ \\
\hline Farmacêutico & 3 & $3,5 \%$ \\
\hline Nutricionista & 3 & $3,5 \%$ \\
\hline Psicólogo & 2 & $2,4 \%$ \\
\hline Educador físico & 1 & $1,2 \%$ \\
\hline Cirurgião dentista & 2 & $2,4 \%$ \\
\hline Assistente Social & 1 & $1,2 \%$ \\
\hline \multicolumn{3}{|l|}{ Turno de trabalho } \\
\hline Manhã & 1 & $1,2 \%$ \\
\hline Noite & 1 & $1,2 \%$ \\
\hline Manhã/tarde & 48 & $56,5 \%$ \\
\hline Manhã, tarde e noite & 34 & $40,0 \%$ \\
\hline Ignorado & 1 & $1,2 \%$ \\
\hline \multicolumn{3}{|l|}{ Considera o trabalho } \\
\hline Leve & 2 & $2,4 \%$ \\
\hline Pouco pesado & 26 & $30,6 \%$ \\
\hline Pesado & 33 & $38,8 \%$ \\
\hline Muito Pesado & 23 & $27,1 \%$ \\
\hline Ignorado & 1 & $1,2 \%$ \\
\hline \multicolumn{3}{|l|}{ Horas de serviço diária } \\
\hline $6-8$ horas & 6 & $7,1 \%$ \\
\hline $8-10$ horas & 13 & $15,3 \%$ \\
\hline$>10$ horas & 65 & $76,5 \%$ \\
\hline Ignorado & 1 & $1,2 \%$ \\
\hline \multicolumn{3}{|c|}{ Costuma levar trabalho para casa } \\
\hline Sim & 73 & $85,9 \%$ \\
\hline Não & 11 & $12,9 \%$ \\
\hline Ignorado & 1 & $1,2 \%$ \\
\hline
\end{tabular}




\begin{tabular}{lll} 
Sim & 78 & $91,8 \%$ \\
Não & 5 & $1,9 \%$ \\
Ignorado & 1 & $1,2 \%$ \\
Sistema & 1 & $1,2 \%$ \\
\hline Aproveita as férias para descansar & & \\
Sim & 69 & $81,2 \%$ \\
Não & 10 & $11,8 \%$ \\
Ignorado & $5,9 \%$ & 5 \\
Sistema & $1,2 \%$ \\
\hline
\end{tabular}

Quanto ao consumo de alimentos, $(64,7 \%)$ dos participantes relataram consumir alimentos tanto processados quanto in natura. No entanto, $(31,8 \%)$ declarou que consome mais alimento in natura. Sobre a forma de preparo dos mesmos, $(36,5 \%)$ relatou preferência por alimentos cozidos, enquanto os assados, grelhados, fritos e ensopados apresentaram os seguintes percentuais $(28,2 \%, 22,4 \%, 10,6 \%$ e $24 \%)$, respectivamente. Dos entrevistados, (80\%) afirmaram não adicionar sal no alimento após estar preparado. O tipo de tempero mais comum foi o natural (52,9\%).

Em relação aos fatores de morbidade referida, $(35,3 \%)$ relatou uso de anticoncepcional/terapia de reposição hormonal. Quanto ao AUDIT, $(90,6 \%)$ dos residentes foram classificados como "Zona I" e (8,25\%) caracterizados como "Zona II". É fundamental levar em consideração fatores como o abuso e dependência de álcool entre os profissionais da área da saúde ${ }^{11}$. Além disso, as características relacionadas ao trabalho podem colaborar para o início dessa problemática, o que causa um alerta para os profissionais que atuam na gestão e formulação de políticas de promoção da saúde do trabalhador.

A tabela 2 representa os dados referentes ao trabalho dos participantes, nota-se que a classe profissional predominante (60\%) era composta por médicos, seguidos dos enfermeiros (20\%). Referente ao local de trabalho, a maioria $(76,5 \%)$ atuava em um Hospital Universitário. No que diz respeito ao turno de trabalho, $(56,5 \%)$ atuavam durante o período da manhã e tarde e $(40 \%)$ em modo interno (manhã, tarde e noite). Desses residentes, $(38,8 \%)$ consideram o trabalho como "pesado", $(30,6 \%)$ classificam o trabalho como "pouco pesado" e $(27,1 \%)$ como trabalho "muito pesado". Referente à jornada de trabalho, (76,5\%) trabalhavam por mais de 10 horas diárias e $(15,3 \%)$ em um período entre 8 e 10 horas.

Os riscos modificáveis são o foco no que diz respeito ao controle e à prevenção. Estes estão comumente associados aos hábitos e ritmos de vida dos indivíduos. Sabendo que com o desenvolvimento as condições de trabalho e rotina requerem um ritmo acelerado, os mesmos estão constantemente expostos a fatores que levam ao desenvolvimento de DCV $^{12}$. Ao serem questionados se levavam trabalho para casa, $(85,9 \%)$ relatou levar trabalho para casa, enquanto $(12,9 \%)$ negou levar. Dos participantes, $(91,8 \%)$ tiram férias anualmente e desses, apenas $(81,2 \%)$ aproveitam esse período para descansar.

Os dados relacionados à morbidade referida pelos entrevistados estão dispostos na Tabela 3. Notase que apenas $(1,2 \%)$ declarou possuir Hipertensão arterial e $100 \%$ não possui Diabetes Mellitus. Casos de colesterol alto estiveram presentes em $(12,9 \%)$ dos participantes.

Tabela 3: Apresentação dos dados referente à morbidade referida, Manaus-AM, 2019

\begin{tabular}{lll}
\hline Variáveis & Total=85 & $\%$ \\
Hipertensão Arterial & $\mathrm{n}$ & $1,2 \%$ \\
Sim & & $98,8 \%$ \\
Não & 1 & \\
Diabetes Mellitus & 84 & \\
\hline
\end{tabular}


Mais da metade dos residentes (56,5\%) referiu serem estressados. Confirmando dados de outra observação $^{13}$, onde residentes apresentaram indicativo de estresse em um nível não saudável no qual os sintomas estão presentes na forma de desgaste físico, desejo de fuga e angústia diariamente. Esses são exemplos de sinalizadores de sofrimento psíquico e fragilidades de saúde geral. A prevalência de Síndrome de Burnout entre médicos residentes provoca preocupação, uma vez que pode estar relacionado ao risco de desenvolver depressão, abandono da carreira e até mesmo diminuição na qualidade de assistência a seus pacientes ${ }^{14}$. Dessa forma, medidas preventivas contra seu desenvolvimento são fundamentais para a redução de sua prevalência.

De acordo com o relato sobre antecedentes familiares, a Hipertensão Arterial foi a mais presente, representando (71,8\%), Diabetes Mellitus foi a segunda mais frequente com $(54,1 \%)$, Colesterol Alto representou (52,9\%), Infarto Agudo do Miocárdio, Acidente Vascular Encefálico e Insuficiência Cardíaca, computaram $(22,4 \%, 18,8 \%$ e 9,4\%), respectivamente. Referente aos familiares acometidos, os pais foram os mais acometidos (73\%), outros membros da família representaram 66\% (Tabela 4).

Tabela 4: Histórico familiar para doenças crônicas e desfechos que acometem o sistema cardiovascular, Manaus-AM, 2019

\begin{tabular}{|c|c|c|c|c|c|c|c|c|c|c|}
\hline \multirow[b]{2}{*}{ Variáveis } & \multicolumn{2}{|c|}{ Total $=94$} & \multicolumn{2}{|l|}{ Pai } & \multicolumn{2}{|c|}{ Mãe } & \multicolumn{2}{|c|}{ Pai e Mãe } & \multicolumn{2}{|c|}{ Outros } \\
\hline & $\mathrm{N}$ & $\%$ & $\mathrm{~N}$ & $\%$ & $\mathrm{n}$ & $\%$ & $\mathrm{~N}$ & $\%$ & $\mathrm{~N}$ & $\%$ \\
\hline \multicolumn{11}{|c|}{ Hipertensão Arterial } \\
\hline Sim & 61 & $71,8 \%$ & 24 & $28,2 \%$ & 12 & $14,1 \%$ & 8 & $9,4 \%$ & 17 & $20,0 \%$ \\
\hline Não & 21 & $24,7 \%$ & - & - & - & - & - & - & - & - \\
\hline Não Sabe & 2 & $2,4 \%$ & - & - & - & - & - & - & - & - \\
\hline Ignorado & 1 & $1,2 \%$ & - & - & - & - & - & - & - & - \\
\hline \multicolumn{11}{|c|}{ Infarto Agudo do Miocárdio } \\
\hline Sim & 19 & $22,4 \%$ & 6 & $7,1 \%$ & - & - & - & - & 13 & $15,3 \%$ \\
\hline Não & 58 & $68,2 \%$ & - & - & - & - & - & - & - & - \\
\hline Não Sabe & 5 & $5,9 \%$ & - & - & - & - & - & - & - & - \\
\hline Ignorado & 3 & $3,6 \%$ & - & - & - & - & - & - & - & - \\
\hline \multicolumn{11}{|c|}{ Acidente Vascular Encefálico } \\
\hline Sim & 16 & $18,8 \%$ & 3 & $3,5 \%$ & 1 & $1,2 \%$ & - & - & 12 & $14,1 \%$ \\
\hline Não & 65 & $76,5 \%$ & - & - & - & - & - & - & - & - \\
\hline Não Sabe & 2 & $2,4 \%$ & - & - & - & - & - & - & - & - \\
\hline Ignorado & 2 & $2,4 \%$ & - & - & - & - & - & - & - & - \\
\hline \multicolumn{11}{|c|}{ Diabetes Mellitus } \\
\hline Sim & 46 & $54,1 \%$ & 15 & $17,7 \%$ & 6 & $7,1 \%$ & 2 & $2,4 \%$ & 23 & $27,1 \%$ \\
\hline Não & 38 & $44,7 \%$ & - & - & - & - & - & - & - & - \\
\hline Não Sabe & 1 & $1,2 \%$ & - & - & - & - & - & - & - & - \\
\hline \multicolumn{11}{|c|}{ Colesterol Alto } \\
\hline Sim & 45 & $52,9 \%$ & 13 & $15,3 \%$ & 13 & $15,3 \%$ & 6 & $7,1 \%$ & 13 & $15,3 \%$ \\
\hline Não & 33 & $38,8 \%$ & - & - & - & - & - & - & - & - \\
\hline Não Sabe & 6 & $7,1 \%$ & - & - & - & - & - & - & - & - \\
\hline Ignorado & 1 & $1,2 \%$ & - & - & - & - & - & - & - & - \\
\hline \multicolumn{11}{|c|}{ Insuficiência Cardíaca } \\
\hline Sim & 8 & $9,4 \%$ & 1 & $1,2 \%$ & 3 & $3,5 \%$ & - & - & 4 & $4,7 \%$ \\
\hline Não & 71 & $83,5 \%$ & - & - & - & - & - & - & - & - \\
\hline Não Sabe & 5 & $5,9 \%$ & - & - & - & - & - & - & - & - \\
\hline Ignorado & 1 & $1,2 \%$ & - & - & - & - & - & - & - & - \\
\hline
\end{tabular}

Quanto aos dados antropométricos, a média da Pressão Arterial foi de $(120,01 \pm 13,68)$, nota-se que mais da metade (56,5\%) tinha o Índice de Massa Corporal (IMC) normal, o sobrepreso foi o segundo mais comum $(29,4 \%)$, a obesidade representou $(10,6 \%)$ dos participantes. Referente a circunferência do pescoço, verifica-se que mais da metade $(76,5 \%)$ estava com esse parâmetro dentro da normalidade. Tal percentual fora observado na circunferência do abdome, uma vez que também estava normal, no entanto, $(11,8 \%)$ estava com um risco elevado e $(9,4 \%)$ com risco muito elevado (Tabela 5$)$ 
Tabela 5: Apresentação dos dados antropométricos dos participantes do estudo, Manaus-AM, 2019

\begin{tabular}{|c|c|c|}
\hline \multirow[b]{2}{*}{ Variáveis } & \multicolumn{2}{|l|}{ Total=85 } \\
\hline & $\mathbf{N}$ & $\%$ \\
\hline \multicolumn{3}{|l|}{ Pressão Arterial } \\
\hline Média $\pm \mathrm{dp}$ & \multicolumn{2}{|l|}{$120,01 \pm 13,68$} \\
\hline \multicolumn{3}{|c|}{ Índice de Massa Corporal } \\
\hline Normal & 48 & $56,5 \%$ \\
\hline Sobrepeso & 25 & $29,4 \%$ \\
\hline Obesidade I & 7 & $8,2 \%$ \\
\hline Obesidade II & 2 & $2,4 \%$ \\
\hline Sistema & 3 & $3,5 \%$ \\
\hline \multicolumn{3}{|c|}{ Circunferência do Pescoço } \\
\hline Normal & 65 & $76,5 \%$ \\
\hline Aumentado & 18 & $21,2 \%$ \\
\hline Sistema & 2 & $2,4 \%$ \\
\hline \multicolumn{3}{|c|}{ Circunferência Abdominal } \\
\hline Normal & 65 & $76,5 \%$ \\
\hline Risco Elevado & 10 & $11,8 \%$ \\
\hline Risco Muito Elevado & 8 & $9,4 \%$ \\
\hline Sistema & 2 & $2,4 \%$ \\
\hline
\end{tabular}

A seguir estão apresentadas algumas variáveis que foram cruzadas com as categorias profissionais investigadas. De acordo com o teste de Shapiro Wilk, a idade não era homogênea $(p<0,0001)$. Assim sendo, usou-se o teste não paramétrico de Kruskal Wallis.

A tabela 6 mostra a relação entre dados antropométricos segundo os profissionais. Observa-se que a maioria $(78,3 \%),(p=0,9225)$ apresentou circunferência do pescoço normal. Referente a circunferência do abdome, $(78,3 \%),(p=0,7595)$ fora classificado como normal. Quanto ao IMC também se observa que o maior percentual encontra-se naqueles cuja classificação foi normal $(58,5 \%), \quad(p=0,9673)$. Buscou-se verificar a média da Pressão Arterial dentro de cada categoria, de acordo com o teste de Shapiro Wilk, a idade não era homogênea $(p<0,0001)$. Assim sendo, usou-se o teste não paramétrico de Kruskal Wallis. Ao teste observa-se uma ausência de significância $(p>0,05)$ entre os profissionais.

Tabela 6: Distribuição dos dados antropométricos segundo a categoria profissional investigada, Manaus-AM, 2019

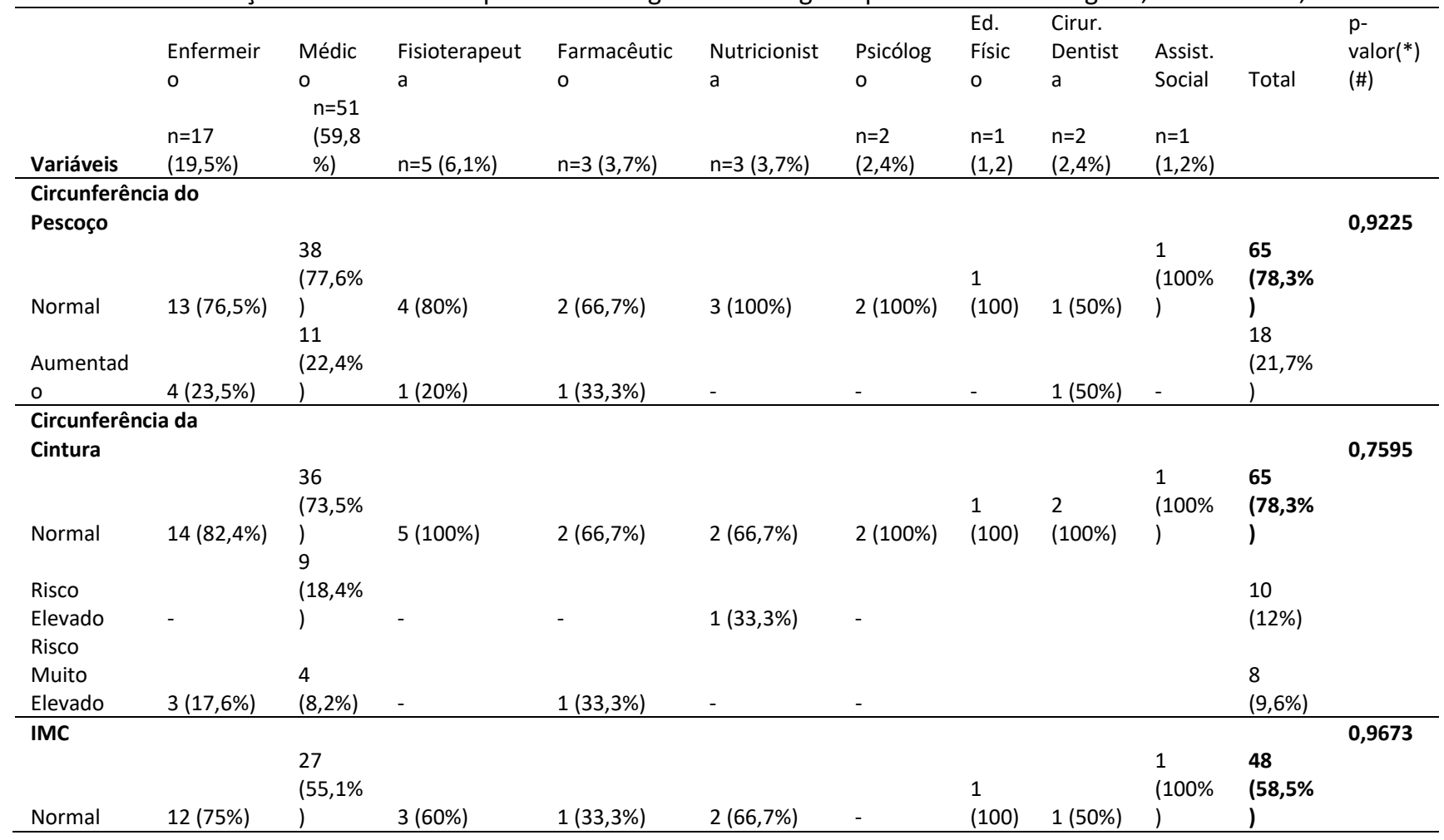




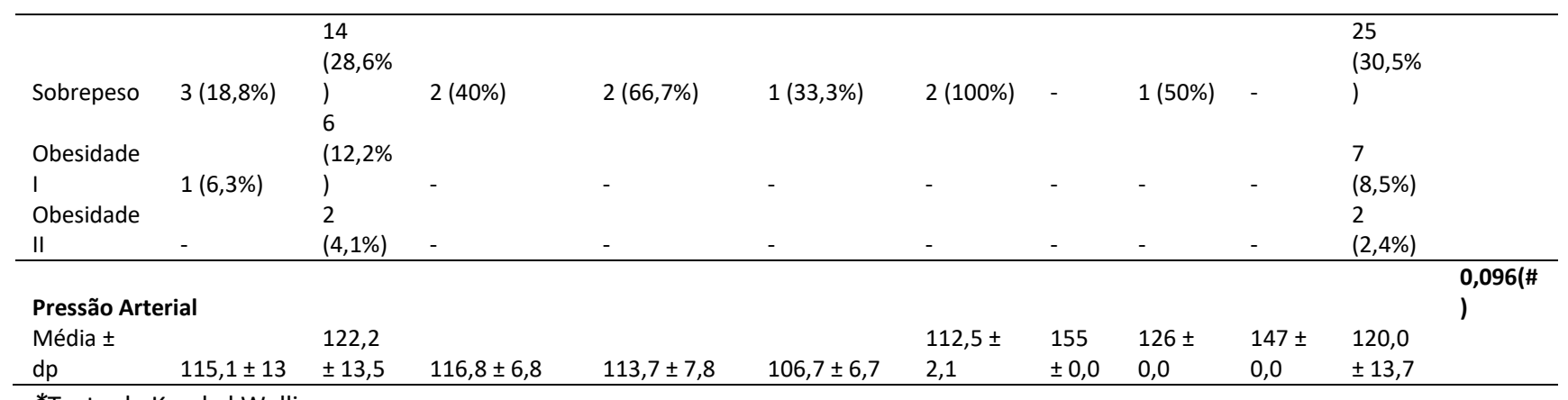

*Teste de Kruskal Wallis

\section{CONCLUSÕES}

Através da identificação de fatores de risco cardiovascular em residentes dos programas de residência multiprofissional e médica de um hospital universitário foram observadas uma série de situações que afetam a saúde desse trabalhador. Uma das observações dos dados foi a recorrência entre os entrevistados relacionada à questão da carga horária de 60 horas semanais de trabalho, associada ao estilo e hábito de vida analisado, o que sobrecarrega a saúde física e emocional dos residentes. Além da verificação de hábitos prejudiciais, como uso abusivo de álcool entre alguns residentes e carga de trabalho excessiva. As distribuições dos dados antropométricos foram feitas de acordo com a categoria profissional investigada e a partir desse levantamento, foram verificadas informações como o sobrepeso, identificado em $30,5 \%$ dos entrevistados, enquanto $8,5 \%$ apresentava obesidade grau 1 e $2,4 \%$ obesidade grau 2 . Além disso, foram verificas as questões relacionadas às medidas de circunferência de cintura e pescoço. Dos participantes, 9,6\% possuíam risco muito elevado para DCV em relação à circunferência da cintura e $21,7 \%$ à circunferência de pescoço.

Através da observação de dados que corroboram para o risco de DCV nessa população, se faz necessário questionar: quem cuida de quem cuida? Logo, faz-se necessária a investigação referente à saúde dos residentes em saúde em mais estudos. A saúde do trabalhador requer atenção por parte dos gestores públicos e hospitalares, uma vez que essa população lida diariamente com demandas de cuidado aos clientes, além das necessidades pessoais.

\section{REFERÊNCIAS}

PEREIRA, F. G. R.; BEZERRA, A. A.; MARQUES, C. C. O.; LUCENA, C. M. F.; SILVA, E. M.; SANTOS, S. F. A.; CANAVIEIRAS, S. A.. Multiprofissionalidade em saúde cardiovascular: atuação integrada em clínica cirúrgica. Revista Brasileira de Ciências da Saúde, v.17, n.3, p.209216, 2013;

BRASIL. Estratégias para o cuidado da pessoa com doença crônica: hipertensão arterial sistêmica. Brasília: Ministério da Saúde, 2013.

SIMÃO, A. F.; PRÉCOMA, D. B.; ANDRADE, J. P.; CORREA FILHO, H.; SARAIVA, J. F. K.; OLIVEIRA, G. M. M.. Primeira diretriz brasileira de prevenção cardiovascular. Arq. Bras. Cardiol., 2013.

MALACHIAS, M. V. B.; SOUZA, W. K. S. B.; PLAVNIK, F. L.; RODRIGUES, C. I. S.; BRANDÃO, A. A.; NEVES, M. F. T.. Sétima diretriz brasileira de hipertensão arterial. Arq. Bras. Cardiol., 2016.

JARDIM, T. V.. Comparação entre fatores de risco cardiovascular em diferentes áreas da saúde num intervalo de vinte anos. 2014.

ORGANIZACIÓN MUNDIAL DE LA SALUD. Monitoreo de avances en materia de las enfermedades no transmisibles 2017. Organización Mundial de la Salud, 2018.

JOMAR, R. T.. Alcohol use disorders identification test (AUDIT) and its applicability in primary health care. Rev. APS, v.15, n.1, p.113-170, 2012.

AGÊNCIA NACIONAL DE SAÚDE SUPLEMENTAR. Manual de diretrizes para o enfretamento da obesidade na saúde suplementar brasileira. Rio de Janeiro: ANS, 2017. 
BIANCHINI, D. C. S.. Tabagismo entre os profissionais de enfermagem de um hospital do interior de minas

gerais. Arquivos de Ciências da Saúde, v.23, n.4, p.37-42, 2016.

FERREIRA, H. S.; LÚCIO, G. M. A.; ASSUNÇÃO, M. L.; SILVA, B. C. V.; OLIVEIRA, J. S.; FLORÊNCIO, T. M. M. T.; GERALDES, A. A. R.; HORTA, B. L.. High blood pressure among students in public and private schools in Maceió, Brazil. 2015.

DINIZ, C. F. G.; ASSUNÇÃO, A. A.; BEINNER, M. A.; PIMENTA, A. M.. Abuso/dependência de álcool e fatores psicossociais do trabalho em profissionais de saúde. Cienc. Cuid. Saude, v.18, n.3, 2019. DOI:

http://dx.doi.org/10.4025/cienccuidsaude.v18i3.45023

COVATTI, C. F.. Fatores de risco para doenças cardiovasculares em adultos e idosos de um hospital universitário. 2016.

CAHU, R. A. G.. Estresse e qualidade de vida em residência multiprofissional em saúde. Rev. Bras. Ter. Cogn., v.10, n. 2, p.76-83, 2014.

BOND, M. M. K.. Prevalência de Burnout entre Médicos Residentes de um Hospital Universitário. Rev. Bras. Educ. Med., v.42, n.3, p.97-107, 2018.

A CBPC - Companhia Brasileira de Produção Científica (CNPJ: 11.221.422/0001-03) detém os direitos materiais desta publicação. Os direitos referem-se à publicação do trabalho em qualquer parte do mundo, incluindo os direitos às renovações, expansões e disseminações da contribuição, bem como outros direitos subsidiários. Todos os trabalhos publicados eletronicamente poderão posteriormente ser publicados em coletâneas impressas sob coordenação da Sustenere Publishing, da Companhia Brasileira de Produção Científica e seus parceiros autorizados. Os (as) autores (as) preservam os direitos autorais, mas não têm permissão para a publicação da contribuição em outro meio, impresso ou digital, em português ou em tradução. 